\title{
Fatal Subarachnoid Hemorrhage From Ruptured Intracerebral Aneurysm After Carotid Endarterectomy
}

\author{
Stephanie Yee ${ }^{\mathrm{a}, \mathrm{b}}$, Manuel Portalatin ${ }^{\mathrm{a}}$, Monica Sridhar ${ }^{\mathrm{a}}$, John Perrone ${ }^{\mathrm{a}}$, \\ Adebayo Adunbarin ${ }^{\mathrm{a}}$, Manrique Guerrero ${ }^{\mathrm{a}}$, John M. Danks ${ }^{\mathrm{a}}$, \\ Jamshed Zuberia ${ }^{\mathrm{a}}$, Alan J. Sori ${ }^{\mathrm{a}}$
}

\begin{abstract}
Concomitant carotid stenosis with cerebral aneurysm is a rare entity with an incidence reported to be about $1.9-3.2 \%$. The effect of carotid revascularization on pre-existing cerebral aneurysm, along with risk factors for aneurysmal rupture, is not fully understood. We report a 61-year-old man who underwent carotid endarterectomy (CEA) for symptomatic carotid stenosis, and 6 days post-operatively the patient suffered a fatal subarachnoid hemorrhage from the rupture of a known basilar artery aneurysm. This rare but potentially fatal complication of a ruptured cerebral aneurysm after CEA warrants a discussion on the management of concomitant pathology.
\end{abstract}

Keywords: Carotid endarterectomy; Revascularization; Aneurysmal rupture; Subarachnoid hemorrhage

\section{Introduction}

The incidence of extracranial internal carotid artery (ICA) stenosis with ipsilateral intracranial aneurysm has been reported to be $1.9-3.2 \%$ [1]. Currently there is level 1 evidence to support carotid endarterectomy (CEA) for symptomatic and asymptomatic patients with critical carotid stenosis; however, the treatment of carotid stenosis in the setting of a pre-existing intracranial aneurysm is undetermined [2]. Due to the infrequency of concomitant carotid stenosis with intracranial aneurysm, the effect of carotid revascularization on the risk of preexisting cerebral aneurysmal rupture remains unclear. Cerebral hyperperfusion syndrome (CHS) is thought to occur in patients after carotid revascularization as blood flow is restored to the brain with underlying impaired cerebral autoregulation due to chronic hypoperfusion from carotid stenosis [3]. The increase

Manuscript submitted December 16, 2019, accepted January 6, 2020

aDepartment of Surgery, St. Joseph's University Medical Center, Paterson, NJ, USA

${ }^{b}$ Corresponding Author: Stephanie Yee, Department of Surgery, St. Joseph's University Medical Center, 703 Main Street, Paterson, NJ 07504, USA.

Email: syee.123@gmail.com

doi: https://doi.org/10.14740/jmc3403 in cerebral perfusion pressure after revascularization is thought to increase aneurysmal wall shear stress, potentially contributing to risk of aneurysmal rupture [4]. We discuss a case of a patient who presented with symptomatic right ICA stenosis with an incidental finding of pre-existing cerebral aneurysm. Six days after an uncomplicated carotid revascularization, the patient developed a subarachnoid hemorrhage (SAH) which shortly after resulted in death.

\section{Case Report}

A 61-year-old man with a history of hypertension, diabetes mellitus, hyperlipidemia, coronary artery disease, chronic kidney disease stage III, and diastolic heart failure (ejection fraction $40 \%$ ) with bioprosthetic aortic valve presented to the hospital with lightheadedness and dark stools. The patient was noted to have anemia with a working diagnosis of gastrointestinal bleeding and was transfused two units of packed red blood cells, which clinically resolved. On hospital day 2, the patient had sudden left hemiplegia and dysmetria which resolved within $4 \mathrm{~h}$ from onset. Vitals on admission were notable for a blood pressure of $147 / 70 \mathrm{~mm} \mathrm{Hg}$ and a heart rate of 88 beats/min. On neurologic examination after the ischemic incident, the patient did not have any residual neurologic deficits. Initial head computed tomography (CT) showed old bilateral infarcts and no acute abnormalities. CT angiogram of head revealed $99 \%$ stenosis of right ICA (Fig. 1a), foci of probable ischemia in the right inferior temporal and posterior parietal lobes, and an incidental aneurysm of the tip of basilar artery measuring $3 \times 5 \times 4 \mathrm{~mm}$ (Fig. 1b). Brain magnetic resonance imaging (MRI) showed no acute infarct. Carotid duplex showed at least $99 \%$ stenosis of right ICA, and about $50 \%$ stenosis of left ICA.

The patient underwent an uncomplicated right CEA with bovine patch angioplasty. Systolic blood pressure (SBP) for majority of case was in the range of $140-160 \mathrm{~mm} \mathrm{Hg}$, with one episode of $200 \mathrm{~mm} \mathrm{Hg}$ requiring single push of $5 \mathrm{mg}$ intravenous (IV) metoprolol. Post-operatively, the patient was transferred to surgical intensive care unit, and was started on aspirin and clopidogrel. SBP was initially high and a nicardipine drip was used to maintain a systolic blood of less than 140 for $24 \mathrm{~h}$. The patient remained without neurologic deficits and was discharged home on post-operative day 1 . Six days later 


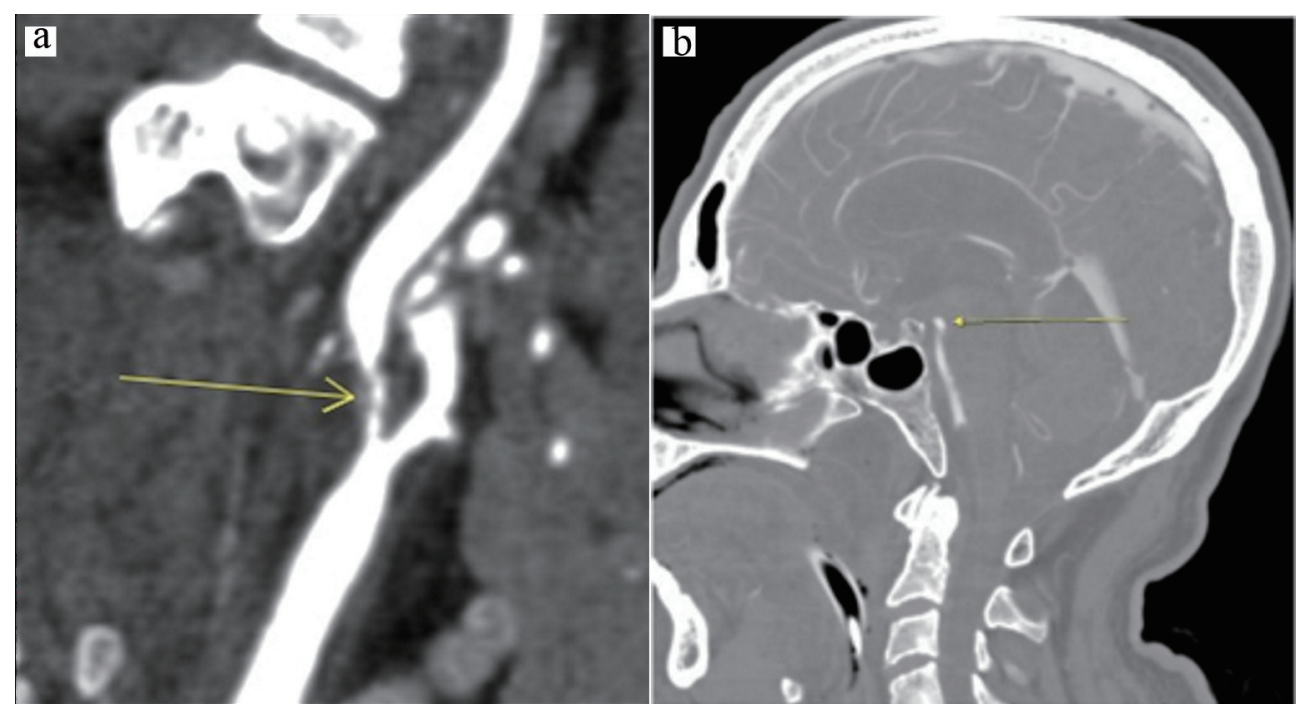

Figure 1. (a) Initial CT angiogram showing $99 \%$ stenosis of right internal carotid artery; (b) Initial CT angiogram showing $3 \times 5 \times$ $4 \mathrm{~mm}$ basilar artery aneurysm. CT: computed tomography.

while at home, the patient had sudden onset of severe headache with loss of consciousness requiring endotracheal intubation. On readmission, the patient was noted to have a blood pressure of 180/80, unequal non-reactive pupils and demonstrated flexion withdrawal of the right arm. Head CT showed diffuse SAH (Fig. 2a), and CT angiogram of head again demonstrated an aneurysm at the tip of basilar artery measuring $3 \times 5$ $\times 4 \mathrm{~mm}$, presumably the source of SAH (Fig. 2b). Bilateral common, internal and external carotid arteries were without significant stenosis. The patient had an extra-ventricular drain placed and was taken emergently for pipeline embolization of the basilar tip and a right superior cerebellar artery aneurysm. He remained intubated post procedure and never regained any neurologic function, developed multi-organ system failure and was terminally extubated on post-operative day 11 .

\section{Discussion}

SAH as a result of ruptured intracranial aneurysm post-carotid intervention has only been reported in five other cases in the current literature [5]. The low incidence of SAH after carotid revascularization was also reported in the North American Symptomatic Carotid Endarterectomy Trial, where only one of 90 patients had ruptured aneurysm after CEA [1]. While the reported incidence of tandem pathology is low, many cases re-
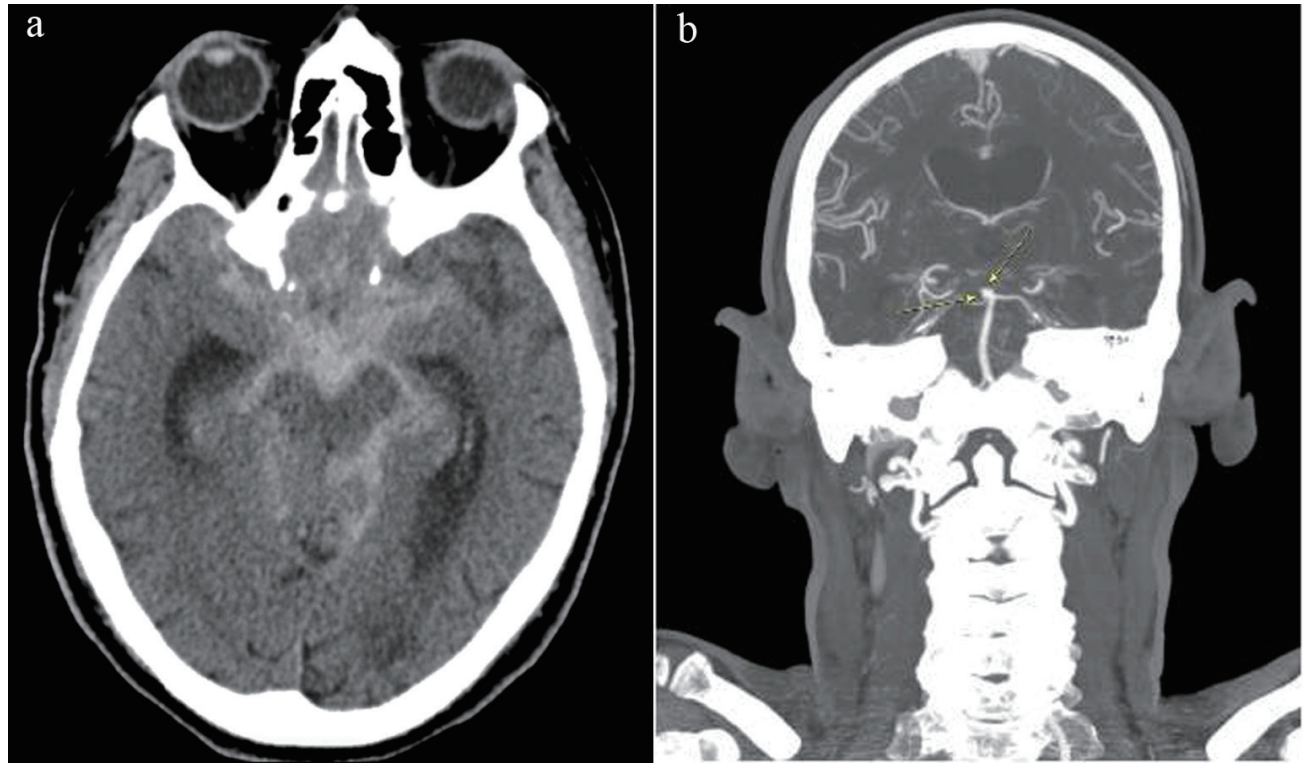

Figure 2. (a) Head CT on post-operative day 6 showing diffuse subarachnoid hemorrhage likely from rupture of basilar artery aneurysm; (b) CT angiogram on post-operative day 6 again showing basilar artery aneurysm. CT: computed tomography. 
main undiagnosed or are only diagnosed on post-mortem analysis. While there is no evidence that carotid revascularization increases the risk of intracranial aneurysmal rupture, it is a potentially fatal phenomenon that warrants further investigation.

The underlying physiological effect of carotid revascularization on an intracranial aneurysm is not well defined; however, certain mechanisms have been proposed that may contribute to its pathophysiology. CHS is a rare but fatal complication that may occur as a result of carotid revascularization and is a mechanism that can help elucidate the physiologic consequences that cause aneurysmal rupture. CHS occurs in $0.2-18.9 \%$ of patients after carotid revascularization [6]. Hyperperfusion is an increase in cerebral blood flow compared to pre-operative values, and CHS most commonly occurs in patients with an increase in cerebral blood flow of greater than $100 \%$, but is rare in patients with less than a $100 \%$ increase [6]. Potential physiologic consequences of CHS include transudation of fluid into pericapillary astrocytes and interstitium leading to vasogenic white matter edema, hyperplasia of endothelial cells, fibrinoid necrosis, extravasation of erythrocytes with petechial hemorrhages and possible intracerebral hemorrhage [7]. The entity of CHS supports the fact that carotid revascularization can disrupt cerebral blood flow and perfusion enough to initiate tissue damage. The brain normally has an autoregulatory mechanism where arterioles dilate or constrict to maintain constant cerebral blood flow and consequently intracranial pressure across a range of mean arterial pressure or other stimuli [8]. In the setting of extracranial carotid stenosis, there is chronic exhaustion of the autoregulatory mechanism because the cerebral arterioles are maximally dilated to compensate for decreased perfusion from the carotid stenosis [8]. Since patients with carotid stenosis are known to have impaired microvascular autoregulation, carotid revascularization can cause hyperperfusion-related damage and the impaired vascular autoregulatory system is unable to accommodate the increased perfusion pressure. The disruption of flow regulation and the increase in wall shear stress in the setting of impaired autoregulation may contribute to increased risk of aneurysmal rupture [9].

Though it may be logical to suspect that carotid revascularization directly affects cerebral flow which may increase the risk of aneurysmal rupture, case reports demonstrate variable outcomes of CEA on cerebral aneurysms $[5,10,11]$. One report demonstrates aneurysmal growth that led to rupture 7 months after CEA. The aneurysm initially measured $5 \times 10$ $\mathrm{mm}$ which then enlarged to $14 \times 10 \mathrm{~mm}$ when discovered on post-mortem analysis [12]. A second case report revealed near complete spontaneous regression of an $8 \mathrm{~mm}$ left anterior communicating artery aneurysm 14 months after CEA [11]. A retrospective cohort study by Yang et al compared the natural history of intracranial aneurysm between patients who had revascularized carotid stenosis versus non-revascularized carotid stenosis. This study included a study cohort of 39 patients, among whom 20 patients $(51.3 \%)$ underwent carotid revascularization with either CEA or carotid stenting, and 19 patients opted for medical management. At the follow-up period of 1.62 years, one patient in each group developed an enlarged posterior circulation aneurysm at follow-up, suggesting the effect of carotid revascularization on aneurysmal size has yet to be defined [13]. It has been suggested that risk factors for aneurysm rupture include architecture, location, and size. Rupture risk for a cerebral aneurysm with $<10 \mathrm{~mm}$ diameter is $0.05 \%$ per year, larger aneurysms $7-12 \mathrm{~mm}$ of anterior circulation have $2.6 \% 5$-year cumulative risk, and giant aneurysms $>25 \mathrm{~mm}$ of posterior circulation have up to a $50 \%$ risk of rupture $[14,15]$. The posterior circulation location is a significant predictor of rupture $[15,16]$. Architecture of the aneurysm such as bleb formation, associated with wall weakness, may also contribute to risk of rupture [16]. The overall risk of aneurysmal rupture after revascularization may depend more on specific aneurysmal growth risk factors than on an increase in cerebral perfusion.

Another important consideration is the indications and appropriate timing for treating incidental cerebral aneurysm in the setting of carotid stenosis. When comparing the natural history of intracranial aneurysms between patients who had undergone carotid revascularization versus those without, no aneurysmal rupture was observed in either group after 1.6 years of follow-up; this particular study suggests that revascularization does not increase risk of aneurysmal rupture [13]. A systematic review by Khan et al reviewed 26 case reports and case series which included a total of 141 patients with tandem pathology from 1965 to 2011. Outcomes in terms of death or stroke rates were analyzed in patients with tandem pathology who had carotid intervention. Many patients had staged interventions: aneurysm clipping followed by CEA, carotid artery stenting followed by aneurysm coiling or CEA followed by aneurysm clipping. This study showed that SAH secondary to aneurysmal rupture occurred only in patients who underwent CEA alone, and there were no reports of SAH in patients who underwent intervention for both the carotid stenosis and cerebral aneurysm [5]. This study suggests that treating both pathologies may reduce risk of aneurysmal rupture; however, the number of patients included in this review was small. The indication for intracranial aneurysmal repair in the setting of carotid stenosis is still unclear, and the question of which pathology to address first still remains unsettled. Given the small but potentially fatal risk of SAH, the consideration of prophylactic aneurysmal repair should be investigated.

\section{Conclusions}

Currently there are no clear guidelines for the management of concomitant carotid stenosis and intracranial aneurysm. The effect of carotid revascularization on aneurysm physiology remains unclear. The risk of potentially fatal aneurysmal rupture after carotid revascularization brings to attention the need for further investigation of risk factors for aneurysmal rupture, and the consideration of pre-operative diagnostic imaging and treatment of intracranial aneurysms in the setting of carotid stenosis.

\section{Acknowledgments}

None to declare. 


\section{Financial Disclosure}

The authors have no disclosure to report.

\section{Conflict of Interest}

None to declare.

\section{Informed Consent}

Not applicable.

\section{Author Contributions}

SY contributed to study concept and design; SY, AA, and JP drafted the manuscript: AJS, JMD, JZ, MP, MG, and MS did the critical revision.

\section{References}

1. Kappelle LJ, Eliasziw M, Fox AJ, Barnett HJ. Small, unruptured intracranial aneurysms and management of symptomatic carotid artery stenosis. North American Symptomatic Carotid Endarterectomy Trial Group. Neurology. 2000;55(2):307-309.

2. Rothwell PM, Eliasziw M, Gutnikov SA, Fox AJ, Taylor DW, Mayberg MR, Warlow CP, et al. Analysis of pooled data from the randomised controlled trials of endarterectomy for symptomatic carotid stenosis. Lancet. 2003;361(9352):107-116.

3. Badruddin A, Taqi MA, Abraham MG, Dani D, Zaidat OO. Neurocritical care of a reperfused brain. Curr Neurol Neurosci Rep. 2011;11(1):104-110.

4. Bouri S, Thapar A, Shalhoub J, Jayasooriya G, Fernando A, Franklin IJ, Davies AH. Hypertension and the post-carotid endarterectomy cerebral hyperperfusion syndrome. Eur J Vasc Endovasc Surg. 2011;41(2):229-237.

5. Khan UA, Thapar A, Shalhoub J, Davies AH. Risk of intracerebral aneurysm rupture during carotid revascularization. J Vasc Surg. 2012;56(6):1739-1747.

6. van Mook WN, Rennenberg RJ, Schurink GW, van
Oostenbrugge RJ, Mess WH, Hofman PA, de Leeuw PW. Cerebral hyperperfusion syndrome. Lancet Neurol. 2005;4(12):877-888.

7. Schwartz RB. Hyperperfusion encephalopathies: hypertensive encephalopathy and related conditions. Neurologist. 2002;8(1):22-34.

8. Sfyroeras G, Karkos CD, Liasidis C, Spyridis C, Dimitriadis AS, Kouskouras K, Gerassimidis TS. The impact of carotid stenting on the hemodynamic parameters and cerebrovascular reactivity of the ipsilateral middle cerebral artery. J Vasc Surg. 2006;44(5):1016-1022; discussion 1022.

9. Hayase H, Tokunaga K, Nakayama T, Sugiu K, Nishida A, Arimitsu S, Hishikawa T, et al. Computational fluid dynamics of carotid arteries after carotid endarterectomy or carotid artery stenting based on postoperative patientspecific computed tomography angiography and ultrasound flow data. Neurosurgery. 2011;68(4):1096-1101; discussion 1101.

10. Siddiqui A, Vora N, Edgell RC, Callison RC, Kitchener J, Alshekhlee A. Rupture of a cerebral aneurysm following carotid endarterectomy. J Neurointerv Surg. 2012;4(5):e27.

11. Li Y, Payner TD, Cohen-Gadol AA. Spontaneous regression of an intracranial aneurysm after carotid endarterectomy. Surg Neurol Int. 2012;3:66.

12. Adams HP, Jr. Carotid stenosis and coexisting ipsilateral intracranial aneurysm. A problem in management. Arch Neurol. 1977;34(8):515-516.

13. Yang W, Rong X, Braileanu M, Jiang B, Garzon-Muvdi T, Caplan JM, Colby GP, et al. Is carotid revascularization safe for patients with concomitant carotid stenosis and intracranial aneurysms? World Neurosurg. 2016;93:1118.

14. Wiebers DO, Whisnant JP, Huston J, 3rd, Meissner I, Brown RD, Jr., Piepgras DG, Forbes GS, et al. Unruptured intracranial aneurysms: natural history, clinical outcome, and risks of surgical and endovascular treatment. Lancet. 2003;362(9378):103-110.

15. International Study of Unruptured Intracranial Aneurysms Investigators. Unruptured intracranial aneurysms - risk of rupture and risks of surgical intervention. N Engl J Med. 1998;339(24):1725-1733.

16. Cebral JR, Sheridan M, Putman CM. Hemodynamics and bleb formation in intracranial aneurysms. AJNR Am J Neuroradiol. 2010;31(2):304-310. 